\title{
Identification of a novel gene regulating amygdala-mediated fear extinction
}

\author{
Ozge Gunduz-Cinar ${ }^{1} \cdot$ Emma Brockway ${ }^{1} \cdot$ Lauren Lederle $^{1} \cdot$ Troy Wilcox $^{2} \cdot$ Lindsay R. Halladay $^{1} \cdot$ Ying Ding $^{3}$. \\ Hyunjung $\mathrm{Oh}^{4,5}$. Erica F. Busch ${ }^{1} \cdot$ Katie Kaugars $^{1} \cdot$ Shaun Flynn $^{1}$ - Aaron Limoges ${ }^{1}$ - Olena Bukalo ${ }^{1}$. \\ Kathryn P. MacPherson ${ }^{1} \cdot$ Sophie Masneuf ${ }^{1} \cdot$ Courtney Pinard $^{1} \cdot$ Etienne Sibille ${ }^{4,5} \cdot$ Elissa J. Chesler $\mathbb{D}^{2}$. \\ Andrew Holmes ${ }^{1}$
}

Received: 3 September 2016 / Revised: 8 October 2017 / Accepted: 30 October 2017 / Published online: 8 January 2018

(c) The Author(s) 2017. This article is published with open access

\begin{abstract}
Recent years have seen advances in our understanding of the neural circuits associated with trauma-related disorders, and the development of relevant assays for these behaviors in rodents. Although inherited factors are known to influence individual differences in risk for these disorders, it has been difficult to identify specific genes that moderate circuit functions to affect trauma-related behaviors. Here, we exploited robust inbred mouse strain differences in Pavlovian fear extinction to uncover quantitative trait loci (QTL) associated with this trait. We found these strain differences to be resistant to developmental crossfostering and associated with anatomical variation in basolateral amygdala (BLA) perineuronal nets, which are developmentally implicated in extinction. Next, by profiling extinction-driven BLA expression of QTL-linked genes, we nominated Ppid (peptidylprolyl isomerase $\mathrm{D}$, a member of the tetratricopeptide repeat (TPR) protein family) as an extinction-related candidate gene. We then showed that Ppid was enriched in excitatory and inhibitory BLA neuronal populations, but at lower levels in the extinction-impaired mouse strain. Using a virus-based approach to directly regulate Ppid function, we demonstrated that downregulating BLA-Ppid impaired extinction, while upregulating BLA-Ppid facilitated extinction and altered in vivo neuronal extinction encoding. Next, we showed that Ppid colocalized with the glucocorticoid receptor (GR) in BLA neurons and found that the extinction-facilitating effects of Ppid upregulation were blocked by a GR antagonist. Collectively, our results identify Ppid as a novel gene involved in regulating extinction via functional actions in the BLA, with possible implications for understanding genetic and pathophysiological mechanisms underlying risk for trauma-related disorders.
\end{abstract}

Electronic supplementary material The online version of this article (https://doi.org/10.1038/s41380-017-0003-3) contains supplementary material, which is available to authorized users.

Ozge Gunduz-Cinar

ozge.gunduzcinar@nih.gov

$\bowtie$ Andrew Holmes

andrew.holmes@nih.gov

1 Laboratory of Behavioral and Genomic Neuroscience, National Institute on Alcohol Abuse and Alcoholism, NIH, Bethesda, MD, USA

2 The Jackson Laboratory, Bar Harbor, ME, USA

3 Joint Carnegie Mellon University-University of Pittsburgh Ph.D. Program in Computational Biology, Pittsburgh, PA, USA

4 Department of Psychiatry, University of Pittsburgh, Pittsburgh, PA, USA

5 Departments of Psychiatry and Pharmacology \& Toxicology, Campbell Family Mental Health Research Institute of CAMH, University of Toronto, Toronto, Canada

\section{Introduction}

There are marked individual differences in risk for traumarelated anxiety disorders including posttraumatic stress disorder (PTSD) [1]. This variation is due in part to the modifying influence of inherited risk and resilience factors and the interaction among genetic variants and stressful life events [2, 3]. Prior studies have uncovered some of the specific genetic candidates involved; finding, for example, increased prevalence of PTSD in trauma-exposed individuals with variants in the pituitary adenylate cyclaseactivating polypeptide, serotonin transporter [4], nociceptin/ orphanin FQ receptor [5], and FK506 binding protein 5 [6] genes, among others [7]. Recent and ongoing genome-wide association studies (GWAS) reveal additional PTSDmoderating genes, including NLGN1, RORA, PRTFDC1, and ANKRD55 [8-11]. However, despite the rapid progress made in delineating neural circuits associated with 
trauma-relevant measures in rodents [12], the genetic factors involved in these behaviors remain poorly understood.

Assaying Pavlovian fear and extinction in rodents has become a widely adopted approach to studying the neural and genetic basis of trauma-related behavior [13-22]. It is becoming increasingly clear that the molecular pathways and neural circuits mediating fear and extinction are shared but also dissociable [12, 13, 21]. Furthermore, previous studies using gene mutation and pharmacological methods, among other techniques, have identified a number of genes, including those mentioned above, that are associated with fear and extinction in humans and rodents alike [5, 19, 23-26]. An alternative approach is to exploit phenotypic and genetic differences across mouse strains to locate genomic regions associated with variation in trauma-relevant behaviors $[16,17]$. This approach has been successfully employed to identify quantitative trait loci (QTL) for conditioned fear [27-38], but has not yet been exploited to examine genetic influences on fear extinction.

The first major aim of the current study was to uncover novel QTL associated with variation in fear extinction in a population generated from a cross between inbred mouse strains (129S1/SvImJ and C57BL/6J, hereafter referred to as 'S1' and 'B6', respectively) that exhibit robust differences in this trait. We then examined developmental factors influencing strain differences in fear extinction, first by crossfostering S1 and B6 mice either prenatally, postnatally, or post-weaning, and then comparing the strains on a neural factor (basolateral amygdala (BLA) perineuronal nets) implicated in the ontogeny of extinction. Next, we performed BLA expression-profiling on genes located within an extinction-associated QTL and, from this analysis, nominated a novel candidate gene, peptidylprolyl isomerase D, Ppid. Then, using a combination of immunocytochemistry and in situ hybridization, we determined the distribution, and neuronal subtype-specific expression of Ppid in the BLA. To establish a causal role for BLA-Ppid in extinction and gain insight into the gene's functional effects on extinction-encoding, we evaluated the effects of viralmediated BLA Ppid upregulation or downregulation on extinction and associated in vivo neuronal activity. Lastly, given Ppid's known role as a hormone receptor modulator, we biochemically determined co-localization of Ppid with the glucocorticoid receptor (GR) in BLA neurons and tested the necessity of GR signaling for the extinction-facilitating effect of Ppid upregulation by pharmacologically antagonizing GR.

\section{Materials and methods}

With the exception of the F1 and F2 populations (which were bred in-house), subjects were adult male C57BL/6J
(B6) and 129S1/SvImJ (S1) mice. The sample size used in each behavioral tests were determined on the basis of findings from our previous fear and extinction studies [39] (see online methods for a full description of all procedures used). For all behavioral and histological procedures, experimenter was blinded to the group of each subject. Following testing, F2 mice were genotyped for QTL mapping, using R/qtl [40]. B6 and S1 mice were cross-fostered at three different developmental time points (prenatal, postnatal, post-weaning) and tested for fear and extinction in adulthood. B6 and S1 mice were examined for BLA (prefrontal cortical and hippocampal) perineuronal nets at various ages, via immunocytochemistry of biotinconjugated lectin wisteria floribunda agglutinin (WFA). BLA perineuronal nets were degraded in S1 mice prior to conditioning by local infusion of chondroitinase $\mathrm{ABC}$ (ChABC), as described for B6 mice [41]. The BLA expression of QTL-located genes was examined following testing of B6 and S1 mice using the Illumina Murine Medium Density Linkage Panel, followed by verification of hits via real-time qPCR in an independently tested cohort. Ppid protein and Ppid gene distribution, localization and quantification in the BLA of B6 and S1 mice were examined using immunohistochemistry, in situ hybridization and real-time qPCR. BLA Ppid expression was manipulated using lentiviral shRNA vectors to knockdown or overexpress the gene, as confirmed by real-time qPCR. In vivo BLA single-unit recordings were performed in S1 mice virally overexpressing Ppid [42, 43]. BLA Ppid and GR colocalization was determined in S1 mice using immunohistochemistry and western blot, and GR expression compared between strain using real-time qPCR and immunohistochemistry. Expression of Hsp90 co-chaperones in S1 mice with BLA Ppid overexpression was determined via realtime qPCR. Effects of the GR agonist, dexamethasone were tested in S1 and B6 mice, and effect of the GR antagonist, RU-486, tested in S1 mice virally overexpressing BLA Ppid.

\section{Results}

\section{Fear and extinction QTL}

We first sought to establish robust extinction differences between two inbred mouse strains to use as a platform for uncovering extinction-related genetic factors. To this end, we showed that the strains exhibited similar freezing during fear conditioning and fear retrieval, but S1 froze significantly more than B6 by the final trial-block of extinction acquisition and on an extinction retrieval test (Fig. 1a). These differences replicate previous reports of a divergent extinction phenotype in the B6 and $\mathrm{S} 1$ inbred strains 


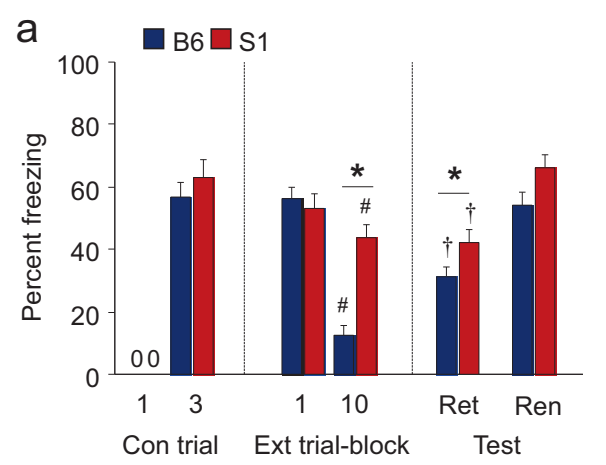

b
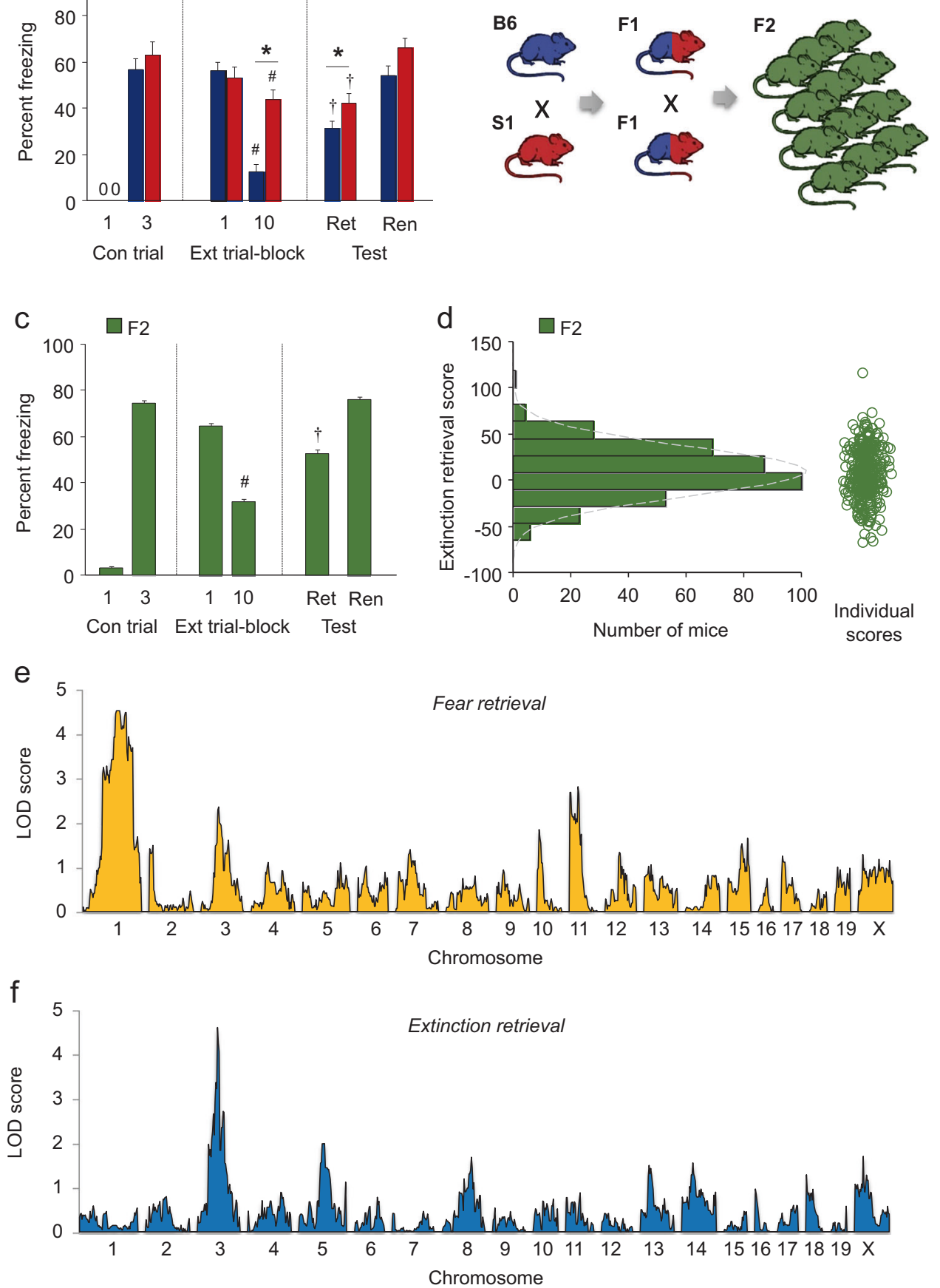

Fig. 1 Genomic loci associated with fear and extinction. a S1 mice showed increased freezing on extinction trial-block $10(t(67)=6.09$, $P<.01, n=31-38)$ and extinction retrieval $(t(67)=1.98, P \leq .05)$, relative to B6 mice. Both strains showed decreased freezing across extinction trial-blocks (B6: $t(30)=11.63, P<.01 ; \mathrm{S} 1: t(37)=2.16, P$ $<.05)$, and on extinction retrieval relative to extinction trial-block 1 (B6: $t(30)=4.75, P<.01 ; \mathrm{S} 1: t(37)=2.25, P<.05)$. b Strategy for producing an F2 population for QTL analysis by intercrossing B6 and S1 mice to produce. $\mathbf{c}$ Decreased freezing across extinction trial-blocks $(t(368)=23.78, P<.01, n=371)$ and on extinction retrieval $(t(368)$ $=8.93, P<.01)$ in F2 mice. d Normal distribution of extinction retrieval scores in the F2 population. Scores calculated as change in freezing from extinction trial-block 1 to extinction retrieval, with negative scores denoting a delta decrease in freezing and positive scores an increase. e Significant genome-wide QTL for fear retrieval (freezing on extinction trial-block 1) on chromosome 1 (4.5 peak LOD, 73.3-182.4 Mb 95\% confidence interval). f Significant genomewide QTL for fear retrieval scores on chromosome 3 (4.6 peak LOD, 77.1-93.1 Mb 95\% confidence interval). ${ }^{*} P<.05 \mathrm{~S} 1$ versus $\mathrm{B} 6,{ }^{\#} P$ $<.05$ Ext trial-block 10 versus $1,{ }^{\dagger} P<.05$ Ret versus Ext trial-block 1 . Data in panels a and $\mathbf{c}$ are means \pm SEM. Con conditioning, Ext extinction, Ret retrieval, Ren renewal 
a

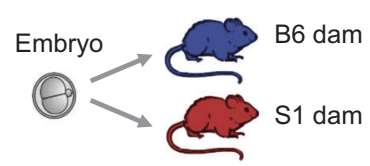

d

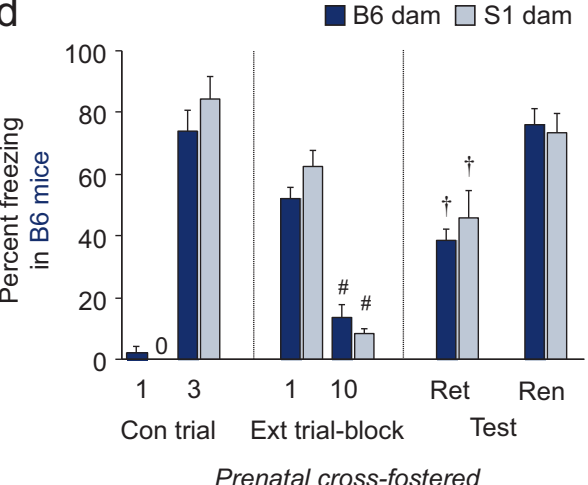

Prenatal cross-fostered

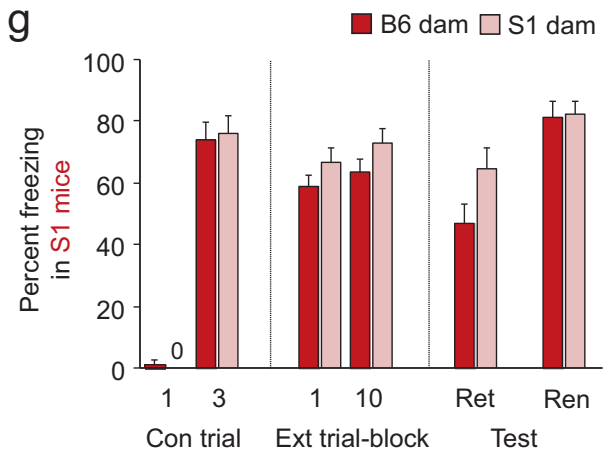

Prenatal cross-fostered

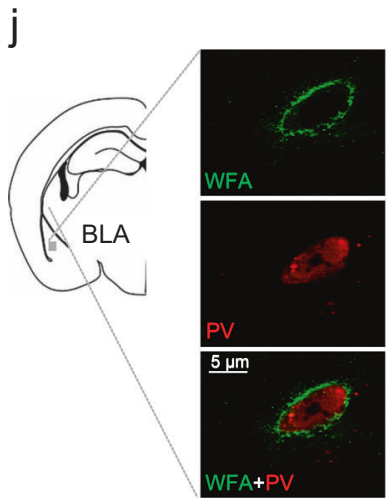

k

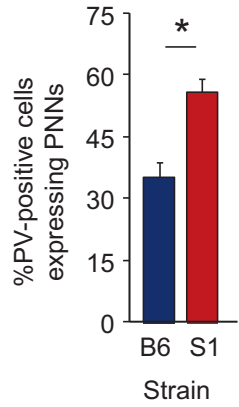

b
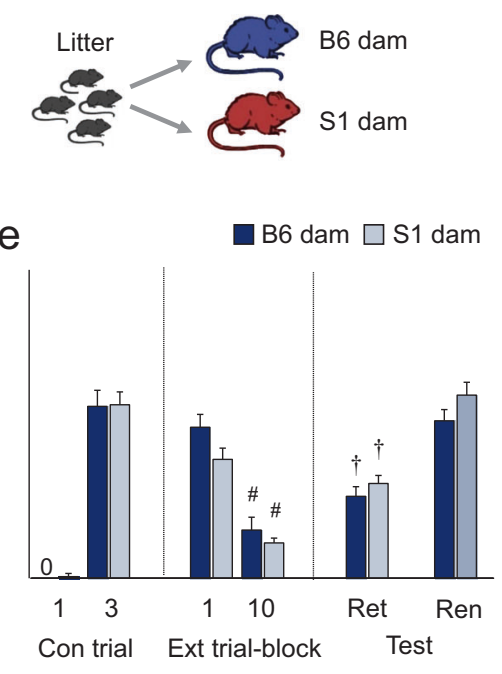

Postnatal cross-fostered

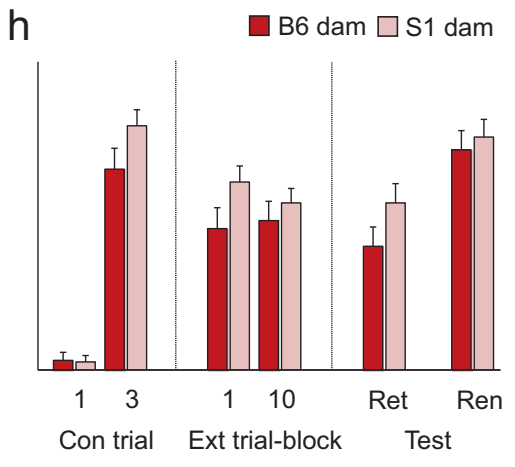

Postnatal cross-fostered
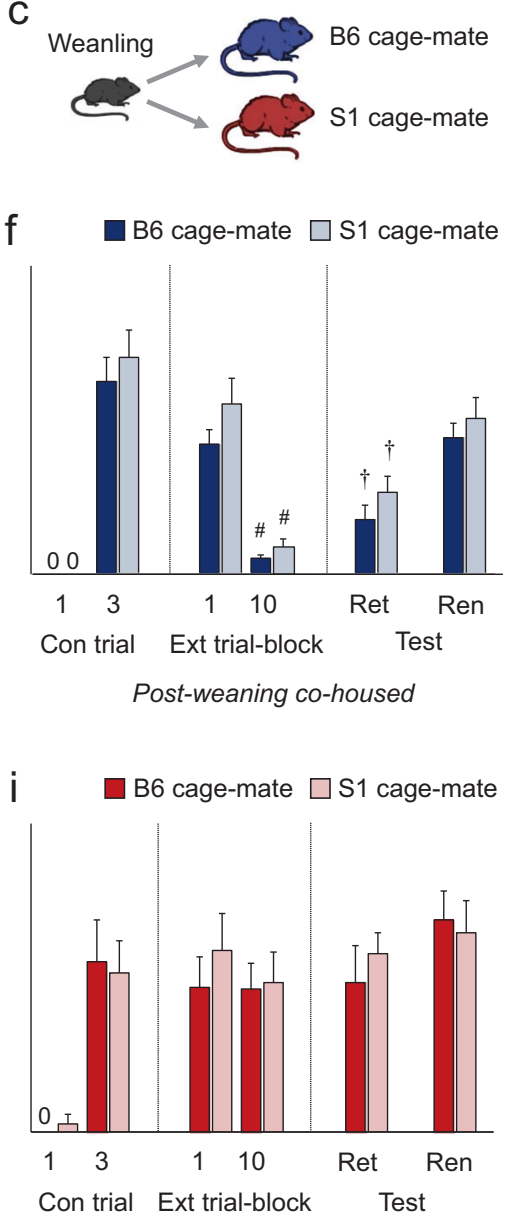

Post-weaning co-housed
I $\mathrm{ChABC} \stackrel{1 d}{\rightarrow} \mathrm{Con} \stackrel{1 d}{\rightarrow} \stackrel{1 d}{\rightarrow}$ Ext $\stackrel{\text { Ret }}{\rightarrow}$

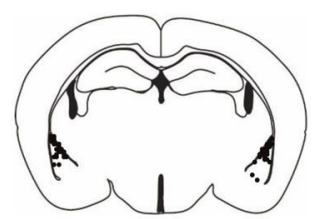

m

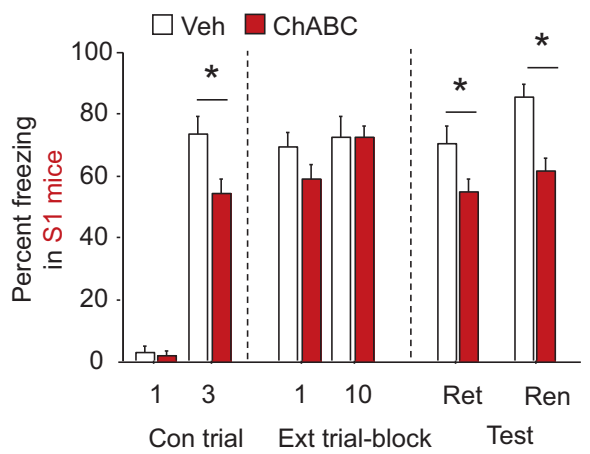

[44-46], consistent with deficient extinction in the S1 strain under the currently employed fear and extinction testing procedures.

Next, to identify sources of genetic variation underlying these phenotypic strain differences, we crossed B6 and S1 mice to derive F1 hybrid mice and, from F1 breeders, generated a F2 population of 371 mice in which each individual mouse carried a unique combination of B6 and
S1 gene variants (Fig. 1b). Assessment of the F2 population (Fig. 1c and d) for fear and extinction behavior revealed, on average, behavior that was more reminiscent of the parental B6, than S1, strain; with a significant decrease in freezing across extinction trial-blocks $(t(368)=23.78, P<.01)$ and significantly lesser freezing on retrieval, relative to extinction trial-block $1(t(370)=8.93, P<.01)($ Figure $\mathrm{S} 1)$. This behavioral profile in the F2 population is suggestive of the 
Fig. 2 Developmental influences on extinction. S1 and B6 were crossfostered a prenatally, b postnatally or $\mathbf{c}$ post-weaning and then tested in adulthood ( $\geq 8$ weeks of age). d Regardless of prenatal cross-fostering, B6 mice showed decreased freezing across extinction trialblocks (B6 dam: $t(9)=12.72, P<.01$; S1 dam: $t(8)=11.33, P<.01$, $n=8-9)$ and on extinction retrieval $(\mathrm{F} 1,17=16.33, P<.01)$. e Regardless of postnatally cross-fostering, B6 mice showed decreased freezing across extinction trial-blocks (B6 dam: $t(22)=6.21, P<.01$; S1 dam: $t(25)=7.30, P<.01, n=22-35)$ and on extinction retrieval (B6 dam: $t(22)=4.783, P<.01$; S1 dam: $t(25)=2.51, P<.05$ ). f Regardless of post-weaning cross-fostering, B6 mice exhibited decreased freezing across extinction trial-blocks (B6 dam: $t(8)=8.47$, $P<.01$; S1 dam: $t(5)=6.44, P<.01, n=5-8)$ and on extinction retrieval (B6 dam: $t(8)=4.14, P<.01$; S1 dam: $t(5)=2.57, P=.05$ ). $\mathrm{S} 1$ mice showed no decrease in freezing across extinction trial-blocks or on extinction retrieval irrespective of prenatal $\mathbf{g}(n=14-16)$, postnatal $\mathbf{h}(n=12-15)$ or post-weaning $\mathbf{i}(n=7-8)$ cross-fostering. $\mathbf{j}$ Representative WFA-labeled PNNs around PV-positive BLA neurons. $\mathbf{k}$ Adult S1 mice had a higher percentage of PV-positive BLA neurons expressing PNNs, relative to B6 mice $(t(136)=4.19, P<.01, n=$ 64-74 sections from $n=10$ mice per strain). The number of overall PV-positive neurons counted did not differ between strains (data not shown). I Cannula placements for intra-BLA ChABC infusions. m Microinfusion of ChABC into the BLA of S1 mice decreased freezing on the final conditioning-trial $(t(36)=2.48, P<.05, n=15-23)$, extinction retrieval $(t(36)=2.22, P<.05, n=15-23)$ and fear renewal (REN) $(t(36)=4.03, P<.01)$, relative to vehicle. $* P<.05 \mathrm{~S} 1$ versus B6, ChABC versus Veh, ${ }^{\#} P<.05$ Ext trial-block 10 versus $1,{ }^{\dagger} P<.05$ Ret versus Ext trial-block 1. Data are means \pm SEM. Con conditioning, Ext extinction, Ret retrieval, Ren renewal

genetic dominance of the B6 allelic effects on extinction. Next, F2s were genotyped using tissue from tail biopsies. Of 1449 SNPs on the linkage panel, 880 were genetically informative (i.e., differed between parental strains) and 778 gave the expected call with parental control DNA. From the genotype data, we performed QTL analysis on four traits: fear retrieval, extinction acquisition, extinction retrieval, and fear renewal (Figure S1, Table S1). We found a QTL at the genome-wide significance level for fear retrieval on chromosome 1 (Fig. 1e) and an independent QTL for extinction retrieval on chromosome 3 (Fig. 1f).

Our finding of a baseline fear-related QTL on chromosome 1 echoes the results of previous fear-QTL studies that have examined other mouse strains [17, 27-34, 36, 47-51], suggesting that this region harbors influential gene variants for this trait. The extinction-related QTL that we identified on chromosome 3, however, is to our knowledge the first example of a genetic locus linked to this trait in rodents. It is also noteworthy that the fear retrieval and extinction retrieval QTL are located at different genomic sites, because it implies independent genetic influences on these traits and thereby provides a novel extension to mounting evidence that fear and extinction are mediated by overlapping but dissociable molecular pathways and neural circuits [12, 13, 21]. Further demonstrating this dissociation, we found that fear retrieval and extinction retrieval was only weakly correlated $(r<.43)$, as was extinction acquisition and extinction retrieval $(r<.37)$. While perhaps not surprisingly, there was a somewhat stronger correlation between fear retrieval and extinction acquisition $(r<.50)$ (Figure S2). Additionally, to statistically test whether the genetic effect on extinction retrieval was independent of extinction acquisition, we evaluated the chromosome 3 QTL effect on extinction retrieval with or without extinction acquisition as a mediating variable. This entailed comparing (1) a 'full' model of extinction retrieval compromising the chromosome 3 genotype + extinction acquisition + error $(-2 \log$-likelihood $=1631.73)$ with (2) a 'reduced' model consisting of only the chromosome 3 genotype $(-2 \log$-likelihood $=1664.49)$. The resultant likelihood ratio test $\left(X^{2}=32, \mathrm{df}=1, P>.05\right)$ suggests that while the extinction retrieval trait was to some extent related to extinction acquisition, this influence is independent of the genetic effect on chromosome 3 and, furthermore, the chromosome 3 extinction retrieval QTL does not have a significant effect on extinction acquisition.

\section{Developmental influences and differences}

Our QTL data indicate that genetic factors contribute, at least in part, to the differences in the fear extinction phenotype in the S1 and B6 strains. They do not, however, discount the potential contribution of environmental factors, particularly during critical periods of development that have been found to shape extinction in rodents and humans $[52,53]$. Therefore, to gain insight into the potential influence of early life environment, we cross-fostered the two strains either prenatally, postnatally or after weaning, and then tested for extinction in adulthood (Fig. 2a-c). Regardless of developmental period, we found that cross-fostering failed to alter the (fear or) extinction phenotype in either strain (e.g., cause a S1-like extinction deficit in fostered B6 mice, or a rescue of extinction in fostered $\mathrm{S} 1$ mice), as compared to control groups cross-fostered to a mouse of the same strain (Fig. 2d-i).

The resistance of S1 mice to cross-fostering implies that the impaired extinction phenotype in this strain may be programmed relatively early in development. Indeed, we confirmed that when tested at a juvenile age ( $\mathrm{P} 17)$ when rats and B6 mice display enhanced extinction ('fear erasure') [54], extinction in S1 mice was already impaired (Figure S3). In the context of these behavioral data, prior work has shown that extinction becomes less effective after weaning, in B6 mice and rats $[41,55]$, coincident with an increase in the number of BLA parvalbumin-positive $(\mathrm{PV}+)$ interneurons surrounded by perineuronal nets (PNNs) [41, 55]. BLA PNNs are comprised of extracellular matrix chondroitin sulfate proteoglycans and posited to emerge during development as a mechanism protecting fear memories from extinction-induced plasticity and synaptic destabilization [41, 55-57]. Interestingly, we found that as 
compared to adult B6 mice, adult S1 mice had a significantly higher number of BLA PV+ interneurons surrounded by PNNs (Fig. 2j-k). Indeed, when we compared the strains across juvenile and adult ages, we found the percentage of $\mathrm{PV}+$ cells surrounded by PNNs was consistently higher in S1 mice in the BLA, but not in subregions of the prefrontal cortex and hippocampus (Figure S4).

We went on to show that locally degrading PNNs via intra-BLA infusion of ChABC in S1 mice reduced freezing (Fig. 2l, m), consistent with a functional role for BLA PNNs in the behavioral phenotype of this strain. It should be noted that while a ChABC-induced reduction in freezing during extinction replicates the effect reported in adult B6 [41], S1 mice also showed a reduction in freezing during the last trial of fear conditioning. This indicates a somewhat differing pattern of ChABC effects in the two strains, with reduced freezing during extinction in the S1 mice potentially reflecting a weakening of the original fear memory, rather than an effect on extinction per se.

Overall, the weight of findings from these cross-fostering and PNN-related experiments lend further credence to the notion that differences in extinction between the B6 and S1 strains may have their origins in development. At the same time, given the ability of PNN degradation to reduce fear in the S1 strain, they illustrate how the extinction phenotype retains some degree of malleability (i.e., 'rescueability') into adulthood.

\section{Nomination of extinction candidates}

We next turned to the question of identifying genetic candidates associated with impaired extinction in the $\mathrm{S} 1$ strain. Our approach was to leverage the results of QTL analysis to examine which of the genes located within the extinctionlinked QTL on chromosome 3 exhibited expression changes in response to extinction testing (Fig. 3a). We again focused on the BLA for this gene expression analysis, given the region's critical role in extinction [22] and evidence of extinction-related BLA abnormalities in S1 mice [58, 59]. Results showed that fear-induced and extinction-induced distinct patterns of BLA gene expression in the two strains, including an overrepresentation of extinction-related QTL genes in S1 mice (Fig. 3b), none of which were upregulated after extinction in B6 mice (Table S2-3).

To isolate which of the extinction-related genes in the S1 strain might represent the most viable candidates for further study, we filtered the expression data to generate a final list of 11 genes that showed a significant $(P<.05)$ $>0.25$-fold increase in expression following extinction, relative to fear (Fig. 3c). The gene exhibiting the biggest expression change after extinction in S1 mice, Ppid (encoding peptidylprolyl isomerase $\mathrm{D}$, also known as cyclophilin 40), also differed in its expression after fear in S1 (downregulated) and B6 (upregulated) mice, indicating that this gene was differentially recruited after extinction and fear in the two strains. We confirmed that Ppid was upregulated in the BLA in response to extinction testing in an independent cohort of S1 mice (Fig. 3d). Furthermore, examination of the genetic sequence of Ppid in the S1 strain, using B6 as the reference, (www.sanger.ac.uk/sa nger/Mouse_SnpViewer), revealed the presence of multiple SNPs and other variants located throughout the gene (Table S4), albeit with a functional significance that remains unclear.

Thus, taken together, these convergent QTL and gene expression findings nominate Ppid as a potential extinction candidate gene.

\section{Ppid distribution and localization in the amygdala}

Our gene expression data demonstrate that Ppid is functionally engaged after extinction in the BLA, but little is currently known about the localization of this gene or its protein product in this brain region. Using immunocytochemistry, we demonstrated that the Ppid protein was densely expressed in the BLA of S1 mice, as well as the adjacent central and basomedial amygdala nuclei (Fig. 3e-f), but that the overall levels of BLA Ppid protein (quantified by western blots) (Figure S3a), as well as mRNA expression (quantified by real-time-qPCR) (Fig. 3i), were significantly lower in $\mathrm{S} 1$, as compared to B6 mice. Using fluorescence-based in situ hybridization, we also found that Ppid was clearly visible in both principal (Slc17a7 (vGlut1) expressing) and inter (Gadl (Gad67) expressing) neurons within the BLA (Fig. 3g-h, Supplementary Figure S5). These anatomical data indicate that Ppid is ideally located to modulate extinction via its expression in the BLA on excitatory and inhibitory neuronal populations, both of which are implicated in extinction [12].

\section{BLA-Ppid knockdown reveals a causal role in extinction}

Next we sought to test for a causal role for Ppid in extinction by examining the effects of BLA-specific viralmediated Ppid knockdown, in both the S1 and B6 strains (Fig. 3j). In B6 mice, Ppid knockdown increased levels of freezing during extinction retrieval, but not other test-phases, as compared to mice transfected with a control virus; i.e., knockdown impaired extinction in the B6 strain (Fig. 3k). We then determined whether knockdown had any demonstrable effects in S1 mice. Remarkably, against the background of the typical, high freezing on extinction retrieval in this strain, Ppid knockdown produced still higher freezing on retrieval, relative to viral controls 
a

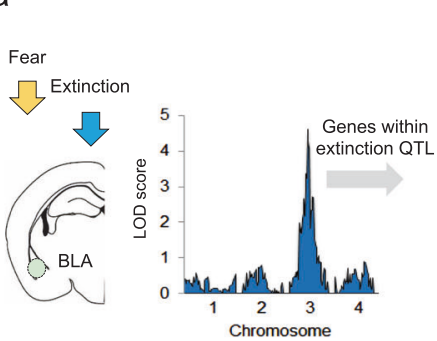

b

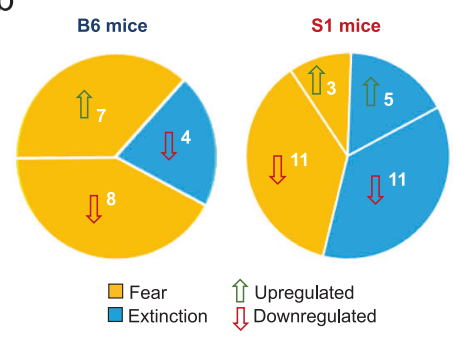

C

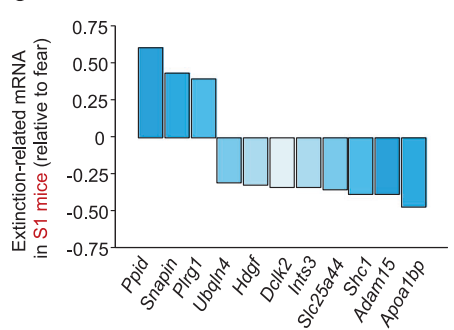

d

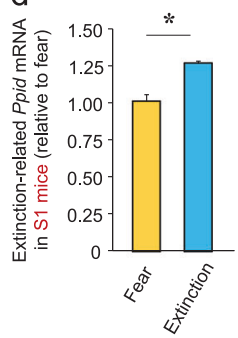

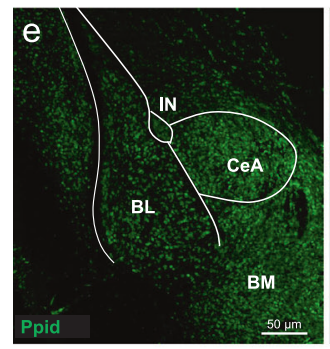
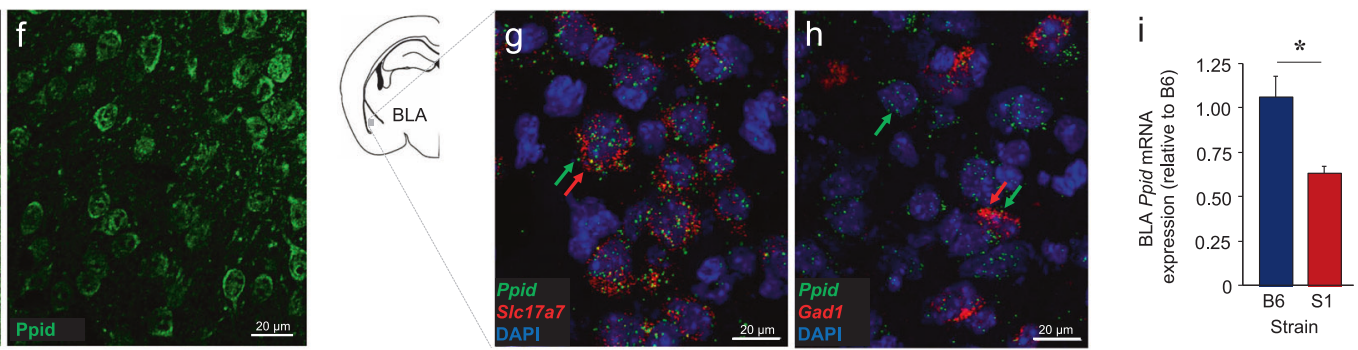

j

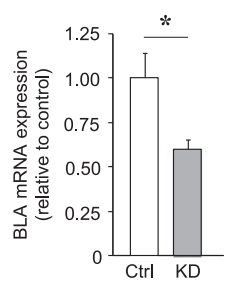

$\mathrm{k}$
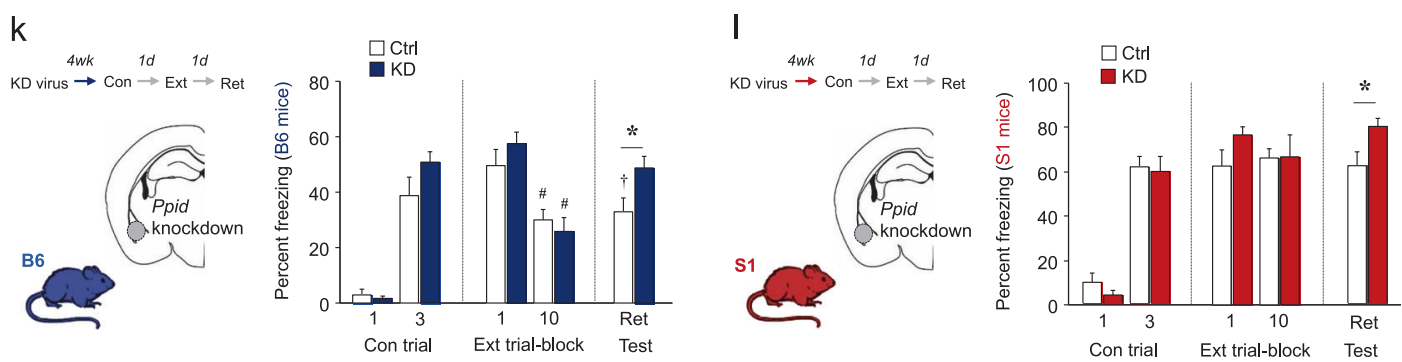

Fig. 3 Ppid nominated as a modulator of amygdala-mediated extinction. a Experimental design for examining differential BLA expression of extinction-QTL genes following fear or extinction. b There were highly divergent fear-related and extinction-related BLA gene expression profiles in S1 and B6 mice (Table S2-3 for full gene lists) $(n=12)$. c Extinction-QTL BLA genes upregulated and downregulated in $\mathrm{S} 1$ mice after extinction, relative to fear. $\mathbf{d}$ Replication of extinction-related Ppid upregulation in the BLA of an independent cohort of S1 mice $(t(9)=5.13, P<.01, n=5-6)$. Ppid protein distribution in BL and surrounding regions $\mathbf{e}$ and in BLA neurons $\mathbf{f}$ of $\mathrm{S} 1$ mice. Ppid mRNA expression (green arrows) on g Slc17a7 (vGlut1, vesicular glutamate transporter 1) labeled principal neurons (red arrows) and on h Gad1 (Gad67, glutamate decarboxylase 67) labeled (red arrows) interneurons in the BLA of S1 mice. i S1 mice had lower Ppid mRNA expression in the BLA, relative to B6 mice $(t(20)=3.54$,

$P<.01, n=11)$. j Viral-mediated Ppid knockdown (KD) in BLA, relative to control virus $(t(25)=2.56, P<.05, n=13-14)$. $\mathbf{k}$ B6 mice with BLA-Ppid KD showed higher freezing on extinction retrieval following, relative to viral controls $(t(13)=2.40, P<.05, n=7-8)$. Freezing on extinction retrieval was lower than on extinction trialblock 1 in the virus-control, but not $\mathrm{KD}$, group $(t(7)=4.59, P<.01)$. The KD and control groups both showed decreased freezing across extinction trial-blocks (Con: $t(7)=3.70, P<.01$; KD: $t(6)=6.62, P$ $<.05)$. I S1 mice with BLA-Ppid KD showed higher freezing on extinction retrieval following, relative to viral controls $(t(17)=2.43$, $P<.05, n=9-10)$. $* P<.05$ Fear versus extinction, S1 versus B6 or Ctrl versus KD. ${ }^{\#} P<.05$ Ext trial-block 10 versus $1,{ }^{\dagger} P<.05$ Ret versus Ext trial-block 1. Data are means \pm SEM. Con conditioning, Ext extinction, Ret retrieval, KD knockdown, Ctrl control.

(Fig. 31). These findings indicate that the loss of Ppid in the BLA is sufficient to disrupt extinction memory formation in B6 mice and exacerbate the already impaired S1 phenotype by incubating fear after repeated CS presentation during extinction training [60, 61]. Importantly, these data also help frame the finding from our gene expression analysis that Ppid was upregulated after extinction in the BLA of S1 mice. Specifically, in light of the extinction-rescuing effects of BLA-Ppid overexpression, the extinction-induced Ppid upregulation in $\mathrm{S} 1$ mice is likely to reflect the engagement of an extinction-facilitating mechanism that is, nonetheless, inadequate ('subthreshold') to rescue the behavioral deficit in these mice in the absence of an additional intervention, such as viral-mediated overexpression (summarized in Figure S11,12).

\section{BLA Ppid upregulation rescues extinction and dampens fear cell activity}

If an extinction-induced increase in BLA Ppid expression in S1 mice remains insufficient to rescue extinction against the background of the abnormally low basal Ppid set-point in these mice, then one prediction is that increasing Ppid levels above a 'functional threshold' might be sufficient. To test this prediction, we examined the consequences of virally overexpressing Ppid (without concomitant alterations in 
expression of other Hsp90-associated genes) in the BLA of S1 mice (Fig. 4a, b). We found that overexpressing Ppid led to a trend for decreased freezing on the final fear conditioning trial and significantly reduced levels of freezing on extinction retrieval, but not other phases of testing, as compared to mice expressing a control virus (Fig. 4c). These data show that, consistent with our prediction, engineering suprathreshold levels of Ppid in the BLA is sufficient to, at least partially, rescue impaired extinction in the S1 strain (summarized in Figure S11,12).

Next, to gain insight into how overexpressing Ppid worked to modulate BLA function to affect extinction, we asked whether the behavioral effects of BLA-Ppid upregulation produced alterations in the neuronal encoding of extinction by recording the activity of BLA neurons during extinction retrieval, in vivo (Fig. 4d). We found that the viral groups did not differ in baseline BLA neuronal firing rate, but that during extinction retrieval a subpopulation of cells exhibited significant changes in firing rate that peaked within $\sim 50 \mathrm{~ms}$ of CS presentation (20 and $11 \%$ of recorded cells in the control and $\mathrm{OE}$ groups, respectively) (Fig. 4e,f). This profile of CSrelated neuronal firing is reminiscent of the BLA 'fear-on cells' previously reported in B6 mice [62]. Entirely in keeping with the persistence of fear in the control-virus S1 mice, CSrelated firing was robust during extinction retrieval (Fig. 4g). By contrast, in S1 mice overexpressing BLA-Ppid, firing of the CS-responsive neuronal population was significantly blunted, consistent with the rescue of extinction produced by Ppid upregulation (Fig. 4g). Thus, these recording data demonstrate that Ppid regulation of extinction has an in vivo correlate in the form of dampened amygdala neuronal encoding of fear-related activity.

\section{Ppid is anatomically and functionally coupled to GR}

In our next set of experiments, we began to probe the potential molecular pathways through which Ppid might regulate extinction. Ppid is a member of the TPR protein family, which along with FKBP5, FKBP52, and PP5, bind heat shock protein 90 (Hsp90) to regulate the function and localization of GRs and other steroid receptors [63, 64]. TPRs are also known to interact with immunosuppressants, such as the calcineurin inhibitor, cyclosporine A (CsA) [65], that have been found to impair fear extinction [66, 67]. However, because little is known about the link between GR and Ppid in the brain, we first asked whether Ppid protein colocalized with GR in the BLA by performing immunostaining and immunoprecipitation. These nonquantitative analyses showed that Ppid-stained neurons exhibited staining for GR (Fig. 4h, i) and that the two protein products co-precipitated in tissue obtained from the BLA (Figure S9).
An anatomical connection between Ppid and GR at the level of the amygdala is particularly intriguing because antagonizing GR in the BLA has been found to impair extinction in rats, whereas administration of a GR agonist, intra-BLA or systemically, facilitates extinction in rats and extinction-deficient populations of B6 mice [7, 68-71]. Moreover, we have previously found evidence of both GR and HPA-axis abnormalities in S1 mice: as compared to B6, $\mathrm{S} 1$ mice exhibit reduced hippocampal $G R$ expression, as well as exaggerated serum corticosterone responses to extinction training and acute swim stress, but blunted corticosterone responses to swim stress after chronic restraint [58]. Here, we extended these observations by showing $G R$ gene expression was significantly lower in the BLA of S1 than B6 mice (Fig. 4j, Supplementary Figure S8b). However, we also found that BLA GR protein expression was not different between strains and, most notably, that preextinction acquisition administration of the synthetic GR agonist, dexamethasone, significantly reduced freezing on drug-free extinction retrieval, relative to vehicle, in S1 mice, but not B6 mice (Fig. 4k, Figure S6). This restriction of dexamethasone's extinction-facilitating effects to the $\mathrm{S} 1$ and not B6 strain is consistent with prior work showing the drug is only effective in subgroups of B6 mice that exhibit poor extinction [71]. On the one hand, these data provide further evidence of abnormal BLA $G R$ gene expression in the S1 strain but, on the other hand, show that activating GR signaling retains the ability to facilitate extinction in these mice to mimic the extinction-rescuing effects of BLA-Ppid overexpression.

Prior work has shown that Ppid interacts with the dynein motor complex to assist translocation of the GR to the nucleus [72-74] and is upregulated in the hippocampus after stress [75]. This suggests that reduced $G R$ expression in the BLA of S1 mice may in part stem from the reduced positive modulation of the gene stemming from the low basal Ppid expression in these mice. Consistent with such a connection, overexpressing Ppid in the BLA led to an increase in $G R$ gene expression in $\mathrm{S} 1$ mice (Fig. 41). This posits a scheme whereby Ppid promotes $G R$-trafficking to trigger transcription of $G R$ and other genes supporting extinction-related plasticity. Finally, providing initial functional support for this model, we found that the ability of BLA-Ppid overexpression to partially restore extinction in S1 mice was prevented by blocking GR signaling with the GR antagonist, RU-486 (Fig. 4m).

Taken together, these findings establish a connection between Ppid and GR and offer a heuristic model for how Ppid might regulate amygdala-mediation extinction by actively coupling with GR to promote the receptor's functional activity and drive extinction-related gene expression in the BLA. 

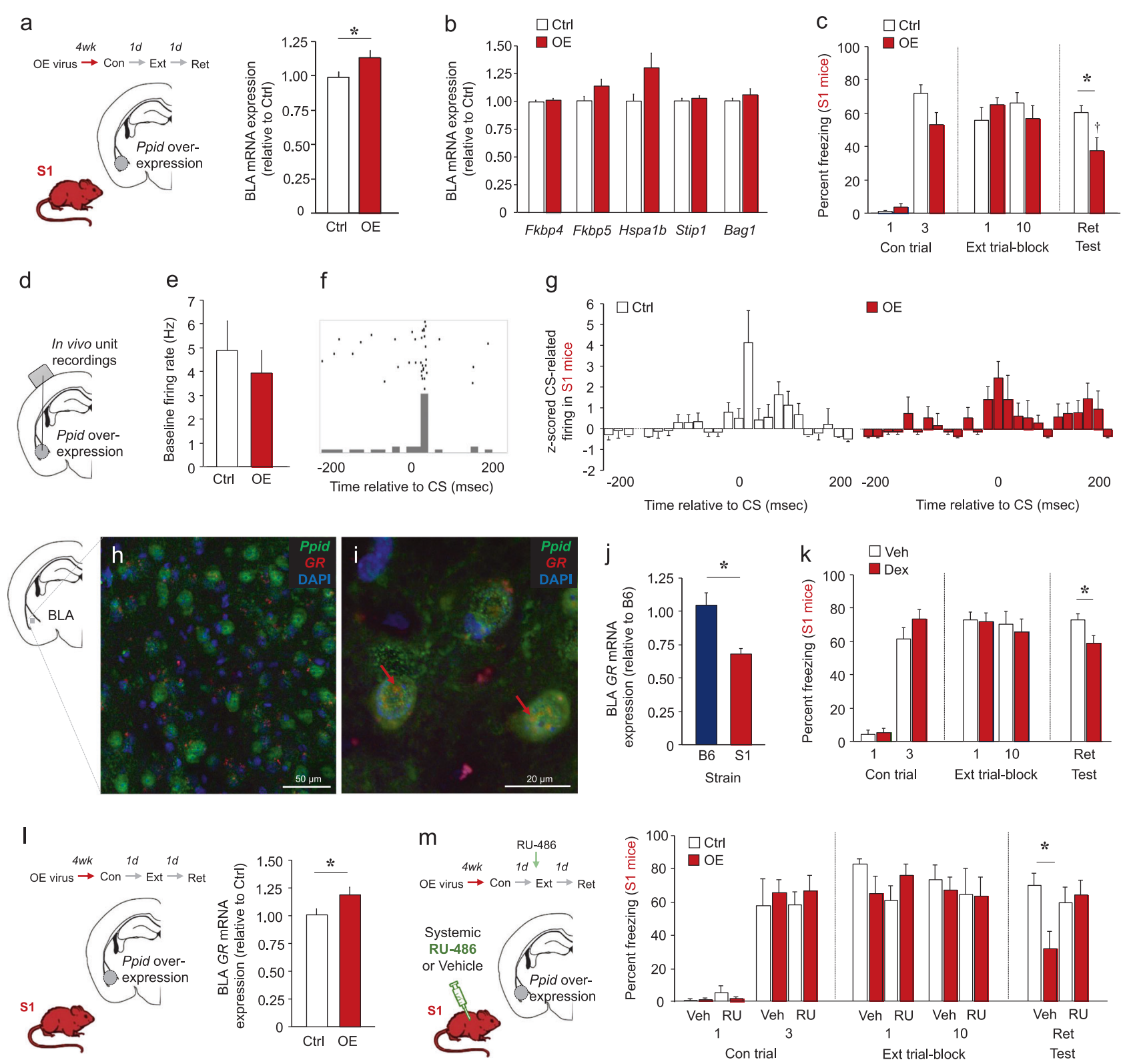

Fig. 4 Ppid regulates amygdala-encoding of extinction and couples to GR. a Viral-mediated Ppid overexpression (OE) (b, but not other Hsp90-associated proteins) in the BLA of S1 mice, relative to control virus $(t(26)=2.25, P<.05, n=13-15)$. c S1 mice with BLA-Ppid OE showed a trend for lower freezing on the final conditioning trial $(t(22)$ $=2.06, P=0.0514, n=12$ ) and significantly lower freezing on extinction retrieval, relative to viral controls $(t(22)=2.47, P<.05, n$ $=12$ ). Freezing on extinction retrieval was lower than on extinction trial-block 1 in the $\mathrm{OE}$, but not control-virus, group $(t(11)=2.85, P$ $<.05)$. d In vivo single-unit recordings were performed in the BLA of Ppid-OE S1 mice during extinction retrieval. e Average baseline firing rate of BLA units did not differ between groups. f Example raster plot of a BLA neurons exhibiting CS-related activity. g The Z-scored activity of CS-related BLA neurons was significantly attenuated during extinction-retrieval in the BLA of Ppid-OE S1 mice, relative to viral controls $(\mathrm{F} 1,390=1.67, P<.01$ group $\times$ time interaction, 116 units from $n=11-12$ mice per group). $\mathbf{h}, \mathbf{i}$ Colocalization of Ppid and GR protein in BLA neurons. j S1 mice showed lower BLA GR mRNA expression, relative to B6 mice $(t(20)=3.67, P<.01, n=11)$. k S1 mice administered the GR agonist, Dex, prior to extinction training showed lower freezing on extinction retrieval, relative to vehicle (Veh) $(t(28)=2.40, P<.05, n=15) .1$ S1 mice with BLA-Ppid OE showed higher BLA GR mRNA expression, relative to viral controls $(t(29)=$ 2.22, $P<.05, n=15-16)$. m S1 mice with BLA-Ppid OE showed lower freezing on extinction retrieval relative to viral-controls if administered Veh, but not the GR antagonist, RU-486 (RU), prior to extinction training $F(3,17)=3.09, P<.05, n=5-6) . * P<.05 \quad$ B6 versus $\mathrm{S} 1$ or Con versus $\mathrm{OE}$ or Veh versus Dex, ${ }^{\dagger} P<.05$ Ret versus Ext trial-block 1. Data are means \pm SEM. Con conditioning, Ext extinction, Ret retrieval, OE overexpression, Ctrl control, Dex Dexamethasone. 


\section{Discussion}

Combining a range of approaches and techniques, the current study reveals a novel genetic locus in mouse that is associated with fear extinction, and demonstrates that Ppid, an amygdala-expressed, GR-associated gene within this locus, plays a causal role in regulating extinction by modulating BLA neuronal encoding.

We found that utilizing QTL analysis proved to be a tractable approach to nominate novel extinction candidates, but it is important to bear in mind that this strategy reveals individual genetic loci that typically only account for a small amount of the variance in a complex trait [76]. As such, our data do not identify Ppid as 'the gene for extinction,' but rather one of many likely modulators of this highly complex behavioral readout. Nonetheless, our findings nominating Ppid are convergent with previous work that has strongly implicated another member of the TPR protein family, Fkbp (FKBP51), as a mediator of stress, fear and extinction in rodents. Given, the human FKBP gene has been implicated as a genetic risk factor for traumarelated disorders [77], it will be interesting to probe for potential links between these disorders and the human PPID gene.

In another parallel with the findings of the current study, these actions of FKBP5 have been linked to effects on amygdala activity and GR function [7, 69, 77, 78]. Interestingly, however, Fkbp binding to Hsp90 generates a Hsp90-GR heterocomplex with lower cortisol affinity and nuclear translocation [77], whereas by promoting nuclei translocation, Ppid acts as a positive modulator of GR [7274]. Given evidence that TPR proteins compete for a single Hsp90-binding site [79], there is likely a process of dynamic interaction between Ppid, FKBP5, and other TRPs that determines how GR signaling is modulated during extinction and other GR-recruiting states [80]. Thus, altering the functional equilibrium of TPRs could represent a novel approach to manipulating GR function for therapeutic goals.

Acknowledgements We are very grateful to Hideko Takahashi and Megan Kopera for their excellent assistance with prenatal crossfostering experiment, and to Javier Rubio and Anna Li for with their generous assistance with the RNAscope experiments, to Jie Liu for valuable discussions and to Tugce Erguven for her assistance. Work supported by the NIAAA Intramural Research Program and NIH Grants AA018776 (EC), DA037927 (EC).

\section{Compliance with ethical standards}

Conflict of interest The authors declare that they have no conflict of interest.

Open Access This article is licensed under a Creative Commons Attribution-NonCommercial-NoDerivatives 4.0 International License, which permits any non-commercial use, sharing, distribution and reproduction in any medium or format, as long as you give appropriate credit to the original author(s) and the source, and provide a link to the
Creative Commons license. You do not have permission under this license to share adapted material derived from this article or parts of it. The images or other third party material in this article are included in the article's Creative Commons license, unless indicated otherwise in a credit line to the material. If material is not included in the article's Creative Commons license and your intended use is not permitted by statutory regulation or exceeds the permitted use, you will need to obtain permission directly from the copyright holder. To view a copy of this license, visit http://creativecommons.org/licenses/by-nc-nd/4.0/.

\section{References}

1. Holmes A, Singewald N. Individual differences in recovery from traumatic fear. Trends Neurosci. 2013;36:23-31.

2. Kendler KS. Twin studies of psychiatric illness: an update. Arch Gen Psychiatry. 2001;58:1005-14.

3. Caspi A, Hariri AR, Holmes A, Uher R, Moffitt TE. Genetic sensitivity to the environment: the case of the serotonin transporter gene and its implications for studying complex diseases and traits. Am J Psychiatry. 2010;167:509-27.

4. Kilpatrick DG, Koenen KC, Ruggiero KJ, Acierno R, Galea S, Resnick HS, et al. The serotonin transporter genotype and social support and moderation of posttraumatic stress disorder and depression in hurricane-exposed adults. Am J Psychiatry. 2007;164:1693-9.

5. Andero R, Brothers SP, Jovanovic T, Chen YT, Salah-Uddin H, Cameron M, et al. Amygdala-dependent fear is regulated by Oprl1 in mice and humans with PTSD. Sci Transl Med. 2013;5:188ra173.

6. Binder EB, Bradley RG, Liu W, Epstein MP, Deveau TC, Mercer $\mathrm{KB}$, et al. Association of FKBP5 polymorphisms and childhood abuse with risk of posttraumatic stress disorder symptoms in adults. Jama. 2008;299:1291-305.

7. Maren S, Holmes A. Stress and fear extinction. Neuropsychopharmacology. 2016;41:58-79.

8. Stein MB, Chen CY, Ursano RJ, Cai T, Gelernter J, Heeringa SG, et al. Genome-wide association studies of posttraumatic stress disorder in 2 cohorts of US army soldiers. JAMA Psychiatry. 2016;73:695-704

9. Kilaru V, Iyer SV, Almli LM, Stevens JS, Lori A, Jovanovic T, et al. Genome-wide gene-based analysis suggests an association between Neuroligin 1 (NLGN1) and post-traumatic stress disorder. Transl Psychiatry. 2016;6:e820.

10. Logue MW, Baldwin C, Guffanti G, Melista E, Wolf EJ, Reardon $\mathrm{AF}$, et al. A genome-wide association study of post-traumatic stress disorder identifies the retinoid-related orphan receptor alpha (RORA) gene as a significant risk locus. Mol Psychiatry. 2013;18:937-42.

11. Xie P, Kranzler HR, Yang C, Zhao H, Farrer LA, Gelernter J. Genome-wide association study identifies new susceptibility loci for posttraumatic stress disorder. Biol Psychiatry. 2013;74:656-63.

12. Tovote P, Fadok JP, Luthi A. Neuronal circuits for fear and anxiety. Nat Rev Neurosci. 2015;16:317-31.

13. Milad MR, Quirk GJ. Fear extinction as a model for translational neuroscience: ten years of progress. Annu Rev Psychol. 2012;63:129-51.

14. Singewald N, Schmuckermair C, Whittle N, Holmes A, Ressler KJ. Pharmacology of cognitive enhancers for exposure-based therapy of fear, anxiety and trauma-related disorders. Pharmacol Ther. 2015;149:150-90.

15. Bukalo O, Pinard CR, Holmes A. Mechanisms to medicines: elucidating neural and molecular substrates of fear extinction to identify novel treatments for anxiety disorders. Br J Pharmacol. $2014 ; 171: 4690-718$ 
16. Flint J, Mackay TF. Genetic architecture of quantitative traits in mice, flies, and humans. Genome Res. 2009;19:723-33.

17. Sokolowska E, Hovatta I. Anxiety genetics-findings from crossspecies genome-wide approaches. Biol Mood Anxiety Disord. 2013;3:9.

18. Milad MR, Pitman RK, Ellis CB, Gold AL, Shin LM, Lasko NB, et al. Neurobiological basis of failure to recall extinction memory in posttraumatic stress disorder. Biol Psychiatry. 2009;66:1075-82.

19. Andero R, Ressler KJ. Fear extinction and BDNF: translating animal models of PTSD to the clinic. Genes Brain Behav. 2012;11:503-12.

20. Griebel G, Holmes A. 50 years of hurdles and hope in anxiolytic drug discovery. Nat Rev Drug Discov. 2013;12:667-87.

21. Orsini CA, Maren S. Neural and cellular mechanisms of fear and extinction memory formation. Neurosci Biobehav Rev. 2012;36:1773-802.

22. Herry C, Ferraguti F, Singewald N, Letzkus JJ, Ehrlich I, Luthi A. Neuronal circuits of fear extinction. Eur $\mathbf{J}$ Neurosci. 2010;31:599-612.

23. Dias BG, Ressler KJ. PACAP and the PAC1 receptor in posttraumatic stress disorder. Neuropsychopharmacology. 2013;38:245-6.

24. Dincheva I, Drysdale AT, Hartley CA, Johnson DC, Jing D, King $\mathrm{EC}$, et al. FAAH genetic variation enhances fronto-amygdala function in mouse and human. Nat Commun. 2015;6:6395.

25. Lee TT, Hill MN, Lee FS. Developmental regulation of fear learning and anxiety behavior by endocannabinoids. Genes Brain Behav. 2016;15:108-24.

26. Hartley CA, McKenna MC, Salman R, Holmes A, Casey BJ, Phelps EA, et al. Serotonin transporter polyadenylation polymorphism modulates the retention of fear extinction memory. Proc Natl Acad Sci USA. 2012;109:5493-8.

27. Parker CC, Sokoloff G, Cheng R, Palmer AA. Genome-wide association for fear conditioning in an advanced intercross mouse line. Behav Genet. 2012;42:437-48.

28. Wehner JM, Radcliffe RA, Rosmann ST, Christensen SC, Rasmussen DL, Fulker DW, et al. Quantitative trait locus analysis of contextual fear conditioning in mice. Nat Genet. 1997; 17:331-4.

29. Sokoloff G, Parker CC, Lim JE, Palmer AA. Anxiety and fear in a cross of $\mathrm{C} 57 \mathrm{BL} / 6 \mathrm{~J}$ and $\mathrm{DBA} / 2 \mathrm{~J}$ mice: mapping overlapping and independent QTL for related traits. Genes Brain Behav. 2011;10:604-14.

30. Talbot CJ, Radcliffe RA, Fullerton J, Hitzemann R, Wehner JM, Flint J. Fine scale mapping of a genetic locus for conditioned fear. Mamm Genome. 2003;14:223-30.

31. Radcliffe RA, Lowe MV, Wehner JM. Confirmation of contextual fear conditioning QTLs by short-term selection. Behav Genet. 2000;30:183-91.

32. Ponder CA, Kliethermes CL, Drew MR, Muller J, Das K, Risbrough VB, et al. Selection for contextual fear conditioning affects anxiety-like behaviors and gene expression. Genes Brain Behav. 2007;6:736-49.

33. Caldarone B, Saavedra C, Tartaglia K, Wehner JM, Dudek BC, Flaherty L. Quantitative trait loci analysis affecting contextual conditioning in mice. Nat Genet. 1997;17:335-7.

34. Owen EH, Christensen SC, Paylor R, Wehner JM. Identification of quantitative trait loci involved in contextual and auditory-cued fear conditioning in BXD recombinant inbred strains. Behav Neurosci. 1997;111:292-300.

35. Parker CC, Sokoloff G, Leung E, Kirkpatrick SL, Palmer AA. A large QTL for fear and anxiety mapped using an F2 cross can be dissected into multiple smaller QTLs. Genes Brain Behav. 2013;12:714-22.
36. Ponder CA, Huded CP, Munoz MB, Gulden FO, Gilliam TC, Palmer AA. Rapid selection response for contextual fear conditioning in a cross between C57BL/6J and A/J: behavioral, QTL and gene expression analysis. Behav Genet. 2008;38:277-91.

37. Park CC, Gale GD, de Jong S, Ghazalpour A, Bennett BJ, Farber $\mathrm{CR}$, et al. Gene networks associated with conditional fear in mice identified using a systems genetics approach. BMC Syst Biol. 2011;5:43.

38. Parker CC, Gopalakrishnan S, Carbonetto P, Gonzales NM, Leung E, Park YJ, et al. Genome-wide association study of behavioral, physiological and gene expression traits in outbred CFW mice. Nat Genet. 2016;48:919-26.

39. Bukalo O, Pinard C, Silverstein S, Brehm C, Hartley N, Whittle $\mathrm{N}$, et al. Prefrontal inputs to the amygdala instruct fear extinction memory formation. Sci Adv. 2015;1:e1500251.

40. Broman KW, Wu H, Sen S, Churchill GA. R/qtl: QTL mapping in experimental crosses. Bioinformatics. 2003;19:889-90.

41. Gogolla N, Caroni P, Luthi A, Herry C. Perineuronal nets protect fear memories from erasure. Science. 2009;325:1258-61.

42. Fitzgerald PJ, Pinard CR, Camp MC, Feyder M, Sah A, Bergstrom HC, et al. Durable fear memories require PSD-95. Mol Psychiatry. 2015;20:901-12.

43. Fitzgerald PJ, Whittle N, Flynn SM, Graybeal C, Pinard CR, Gunduz-Cinar O, et al. Prefrontal single-unit firing associated with deficient extinction in mice. Neurobiol Learn Mem. 2014;113:69-81.

44. Sartori SB, Maurer V, Murphy C, Schmuckermair C, Muigg P, Neumann ID et al. Combined neuropeptide $\mathrm{S}$ and D-cycloserine augmentation prevents the return of fear in extinction-impaired rodents: advantage of dual versus single drug approaches. Int J Neuropsychopharmacol 2016;19:1-11.

45. Hefner K, Whittle N, Juhasz J, Norcross M, Karlsson RM, Saksida LM, et al. Impaired fear extinction learning and corticoamygdala circuit abnormalities in a common genetic mouse strain. J Neurosci. 2008;28:8074-85.

46. Flores A, Valls-Comamala V, Costa G, Saravia R, Maldonado R, Berrendero $F$. The hypocretin/orexin system mediates the extinction of fear memories. Neuropsychopharmacology. 2014;39:2732-41.

47. Mozhui K, Ciobanu DC, Schikorski T, Wang X, Lu L, Williams RW. Dissection of a QTL hotspot on mouse distal chromosome 1 that modulates neurobehavioral phenotypes and gene expression. PLoS Genet. 2008;4:e1000260.

48. Turri MG, Talbot CJ, Radcliffe RA, Wehner JM, Flint J. Highresolution mapping of quantitative trait loci for emotionality in selected strains of mice. Mamm Genome. 1999;10:1098-101.

49. Gershenfeld HK, Paul SM. Mapping quantitative trait loci for fear-like behaviors in mice. Genomics. 1997;46:1-8.

50. Henderson ND, Turri MG, DeFries JC, Flint J. QTL analysis of multiple behavioral measures of anxiety in mice. Behav Genet. 2004;34:267-93.

51. Cook MN, Baker JA, Heldt SA, Williams RW, Hamre KM, Lu L. Identification of candidate genes that underlie the QTL on chromosome 1 that mediates genetic differences in stress-ethanol interactions. Physiol Genom. 2015;47:308-17.

52. Callaghan BL, Graham BM, Li S, Richardson R. From resilience to vulnerability: mechanistic insights into the effects of stress on transitions in critical period plasticity. Front Psychiatry. 2013;4:90.

53. Champagne FA. Epigenetics and developmental plasticity across species. Dev Psychobiol. 2013;55:33-41.

54. Cowan CS, Callaghan BL, Kan JM, Richardson R. The lasting impact of early-life adversity on individuals and their descendants: potential mechanisms and hope for intervention. Genes Brain Behav. 2016;15:155-68. 
55. Callaghan BL, Richardson R. The effect of adverse rearing environments on persistent memories in young rats: removing the brakes on infant fear memories. Transl Psychiatry. 2012;2:e138.

56. Karpova NN, Pickenhagen A, Lindholm J, Tiraboschi E, Kulesskaya N, Agustsdottir A, et al. Fear erasure in mice requires synergy between antidepressant drugs and extinction training. Science. 2012;334:1731-4.

57. Umemori J, Winkel F, Castren E, Karpova NN. Distinct effects of perinatal exposure to fluoxetine or methylmercury on parvalbumin and perineuronal nets, the markers of critical periods in brain development. Int J Dev Neurosci. 2015;44:55-64.

58. Camp MC, Macpherson KP, Lederle L, Graybeal C, Gaburro S, Debrouse LM, et al. Genetic strain differences in learned fear inhibition associated with variation in neuroendocrine, autonomic, and amygdala dendritic phenotypes. Neuropsychopharmacology. 2012;37:1534-47.

59. Gunduz-Cinar O, Macpherson KP, Cinar R, Gamble-George J, Sugden K, Williams B, et al. Convergent translational evidence of a role for anandamide in amygdala-mediated fear extinction, threat processing and stress-reactivity. Mol Psychiatry. 2013;18:813-23.

60. Cain CK, Blouin AM, Barad M. Temporally massed CS presentations generate more fear extinction than spaced presentations. J Exp Psychol. 2003;29:323-33.

61. Macpherson K, Whittle N, Camp M, Gunduz-Cinar O, Singewald $\mathrm{N}$, Holmes A. Temporal factors in the extinction of fear in inbred mouse strains differing in extinction efficacy. Biol Mood Anxiety Disord. 2013;3:13.

62. Herry C, Ciocchi S, Senn V, Demmou L, Muller C, Luthi A. Switching on and off fear by distinct neuronal circuits. Nature. 2008;454:600-6.

63. Ratajczak T, Carrello A, Mark PJ, Warner BJ, Simpson RJ, Moritz $\mathrm{RL}$, et al. The cyclophilin component of the unactivated estrogen receptor contains a tetratricopeptide repeat domain and shares identity withp59 (FKBP59). J Biol Chem. 1993;268:13187-92.

64. Sanchez ER. Chaperoning steroidal physiology: lessons from mouse genetic models of Hsp90 and its cochaperones. Biochim Biophys Acta. 2012;1823:722-9.

65. Hamilton GS, Steiner JP. Immunophilins: beyond immunosuppression. J Med Chem. 1998;41:5119-43.

66. Almeida-Correa S, Moulin TC, Carneiro CF, Goncalves MM, Junqueira LS, Amaral OB. Calcineurin inhibition blocks within-, but not between-session fear extinction in mice. Learn Mem. 2015;22:159-69.

67. de Carvalho Myskiw J, Furini CR, Schmidt B, Ferreira F, Izquierdo I. Extinction learning, which consists of the inhibition of retrieval, can be learned without retrieval. Proc Natl Acad Sci USA. 2015;112:E230-233.

68. Yang YL, Chao PK, Lu KT. Systemic and intra-amygdala administration of glucocorticoid agonist and antagonist modulate extinction of conditioned fear. Neuropsychopharmacology. 2006;31:912-24.

69. Sawamura T, Klengel T, Armario A, Jovanovic T, Norrholm SD, Ressler KJ, et al. Dexamethasone treatment leads to enhanced fear extinction and dynamic Fkbp5 regulation in amygdala. Neuropsychopharmacology. 2016;41:832-46.

70. Ninomiya EM, Martynhak BJ, Zanoveli JM, Correia D, da Cunha C, Andreatini R. Spironolactone and low-dose dexamethasone enhance extinction of contextual fear conditioning. Prog Neuropsychopharmacol Biol Psychiatry. 2010;34:1229-35.

71. Galatzer-Levy IR, Andero R, Sawamura T, Jovanovic T, Papini S, Ressler KJ, et al. A cross species study of heterogeneity in fear extinction learning in relation to FKBP5 variation and expression: implications for the acute treatment of posttraumatic stress disorder. Neuropharmacology. 2017;116:188-95.

72. Galigniana MD, Harrell JM, O'Hagen HM, Ljungman M, Pratt WB. Hsp90-binding immunophilins link p53 to dynein during p53 transport to the nucleus. J Biol Chem. 2004;279:22483-9.

73. Ratajczak T, Ward BK, Minchin RF. Immunophilin chaperones in steroid receptor signalling. Curr Top Med Chem. 2003;3:1348-57.

74. Renoir JM, Mercier-Bodard C, Hoffmann K, Le Bihan S, Ning YM, Sanchez ER, et al. Cyclosporin A potentiates the dexamethasone-induced mouse mammary tumor viruschloramphenicol acetyltransferase activity in LMCAT cells: a possible role for different heat shock protein-binding immunophilins in glucocorticosteroid receptor-mediated gene expression. Proc Natl Acad Sci USA. 1995;92:4977-81.

75. Bourke CH, Raees MQ, Malviya S, Bradburn CA, Binder EB, Neigh GN. Glucocorticoid sensitizers Bag1 and Ppid are regulated by adolescent stress in a sex-dependent manner. Psychoneuroendocrinology. 2013;38:84-93.

76. Mackay TF. The genetic architecture of quantitative traits. Annu Rev Genet. 2001;35:303-39.

77. Zannas AS, Binder EB. Gene-environment interactions at the FKBP5 locus: sensitive periods, mechanisms and pleiotropism. Genes Brain Behav. 2014;13:25-37.

78. White MG, Bogdan R, Fisher PM, Munoz KE, Williamson DE, Hariri AR. FKBP5 and emotional neglect interact to predict individual differences in amygdala reactivity. Genes Brain Behav. 2012;11:869-78.

79. Silverstein AM, Galigniana MD, Chen MS, Owens-Grillo JK, Chinkers M, Pratt WB. Protein phosphatase 5 is a major component of glucocorticoid receptor.hsp90 complexes with properties of an FK506-binding immunophilin. J Biol Chem. 1997;272:16224-30.

80. Kaouane N, Porte Y, Vallée M, Brayda-Bruno L, Mons N, Calandreau L, Marighetto A, Piazza PV, Desmedt A. Glucocorticoids can induce PTSD-like memory impairments in mice. Science. 2012;335:1510-3. 